\title{
Recent BABAR measurements of hadronic $B$ branching fractions
}

\author{
Alessandro GAZ* \\ University of Colorado \\ E-mail: gaz@slac.stanford.edu
}

We present recent $B A B A R$ measurements of branching fractions of $B$ mesons to final states containing at least one charmed hadron. These decays are useful to test our knowledge on the CKM Matrix paradigm and verify the predictions provided by different theoretical frameworks. Moreover, the decays of $B$ mesons to baryons are still poorly understood, so measuring as many final states as possible may lead to the development of more reliable phenomenological models. All results have been obtained using the final $B A B A R$ dataset, which consists of $\sim 467$ million $B \bar{B}$ pairs.

The 2011 Europhysics Conference on High Energy Physics-HEP 2011,

July 21-27, 2011

Grenoble, Rhône-Alpes France

\footnotetext{
* Speaker.
} 


\section{Common analysis tools}

In all the analyses presented here it is possible to fully reconstruct the hadronic final state. Two (almost completely uncorrelated) variables, exploiting the very precise knowledge of the kinematics in a $e^{+} e^{-}$collider, are used to separate signal events from the combinatorial background: the energy-substituted mass of the $B$ meson candidate $m_{\mathrm{ES}} \equiv \sqrt{s / 4-\mathbf{p}_{B}^{2}}$ and the difference between the energy of the reconstructed $B$ candidate and the expected one: $\Delta E \equiv E_{B}-\sqrt{s} / 2$ ( $\sqrt{s}$ represents the energy of the $e^{+} e^{-}$system in the center of mass frame). A significant source of background comes from $e^{+} e^{-} \rightarrow q \bar{q}(q=u, d, s, c)$ events, which typically exhibit a jet-like topology, opposed to the more spherical $B$ decays. This background can be effectively suppressed combining several variables sensitive to the event shape by means of a Fisher discriminant or a Neural Network.

\section{Final states containing a $D^{(*)}$ meson}

The decays of $B$ mesons to final states containing at least one $D^{(*)}$ meson are useful to probe very suppressed amplitudes and in general verify the patterns predicted by different theoretical frameworks.

The interest of the $B^{+} \rightarrow D^{+} K^{(*) 0}$ decays consists in the fact that neither of the (anti)quarks present in the $B$ meson appear in the final state, thus these decays must proceed through annihilation diagrams. The branching fractions are expected to be of the order of $10^{-6}$, but rescattering, particularly through the $D_{s} \pi^{0}$ channel, may play a significant role, enhancing them by almost one order of magnitude. We reconstruct candidate signal events combining $K_{S}^{0} \rightarrow \pi^{+} \pi^{-}, K^{* 0} \rightarrow K^{+} \pi^{-}$, and $D^{+} \rightarrow K^{-} \pi^{+} \pi^{+}, K_{S}^{0} \pi^{+}, K^{-} \pi^{+} \pi^{+} \pi^{0}, K_{S}^{0} \pi^{+} \pi^{0}$ candidates. $B^{+} \rightarrow D^{+} \rho^{0}$ decays constitute a source of peaking background, which is suppressed with a cut on the helicity of the $\pi^{+} \pi^{-}$system. The signal yield is extracted through a maximum likelihood (ML) fit on the $m_{\mathrm{ES}}$ and Fisher variables. No significant signal is found (see e.g. left plot of Figure 1), and we derive the upper limits (at $90 \%$ $\mathrm{CL}): \mathscr{B}\left(B^{+} \rightarrow D^{+} K^{0}\right)<2.9 \times 10^{-6}, \mathscr{B}\left(B^{+} \rightarrow D^{+} K^{* 0}\right)<3.0 \times 10^{-6}$, which are the most stringent limits set to date [1].

The decays $B \rightarrow D^{(*)} D^{(*)} K$ have sizable branching fractions, adding up to a few percent. Several amplitudes contribute to the 22 sub-modes, so these decays offer a unique opportunity to verify in a coherent way the pattern predicted by different theoretical models. From the experimental point of view, one of the main challenges of the analysis is to properly take into account the crossfeed (mostly generated by failing to reconstruct the soft pion from the decays of the $D^{*}$ ) among the different submodes. In that respect, the signal yields are extracted from a ML fit to the $m_{\mathrm{ES}}$ variable with an iterative procedure, in which the branching fractions of the crossfeed modes for the channel under study are taken from the previous iteration of the fit. This procedure ensures that all the 22 submodes are treated in a consistent way. Depending on the particular mode, the signal significance varies between 2.2 and 13.4 standard deviations [2]. No evidence of breaking of the isospin symmetry has been found. Summing separately the charged and the neutral modes, we obtain:

$$
\begin{aligned}
& \mathscr{B}\left(B^{0} \rightarrow D^{(*)} D^{(*)} K\right)=(3.68 \pm 0.10 \pm 0.24) \% \\
& \mathscr{B}\left(B^{+} \rightarrow D^{(*)} D^{(*)} K\right)=(4.05 \pm 0.11 \pm 0.28) \%
\end{aligned}
$$


The decays $B^{0} \rightarrow D^{(*) 0} h^{0}$ (where $h^{0}=\pi^{0}, \omega, \eta, \eta^{\prime}$ ) are color suppressed, and thus particularly suited to show effects from non-factorizable contributions. Different theoretical predictions are available for these modes; in particular SCET predicts that the ratio $R \equiv \mathscr{B}\left(B \rightarrow D^{* 0} h^{0}\right) / \mathscr{B}(B \rightarrow$ $D^{0} h^{0}$ ) is $\sim 1$ ( $\omega$ modes excluded), and HQET computes that the longitudinal polarization fraction $f_{L}$ for the $D^{* 0} \omega$ mode be $\sim 90 \%$. The $B^{-} \rightarrow D^{(*) 0} \rho^{-}$channel (which also constitutes a source of peaking background for the analysis) is widely used as a control sample to calibrate the detector response. The signal yield is extracted through a ML fit to the $\Delta E$ variable and again the crossfeed among the different submodes is estimated using an iterative procedure. The results [3] are in good agreement with the previous measurements and show significant disagreement with predictions based on QCD factorization. We confirm the SCET prediction on the ratio $R$ being consistent with 1 (see right plot of Figure 1) and the measurement of $f_{L}=(66.5 \pm 4.7 \pm 1.5) \%$ for $D^{* 0} \omega$, in poor agreement with HQET predictions, may give an indication of sizable long-range contributions.
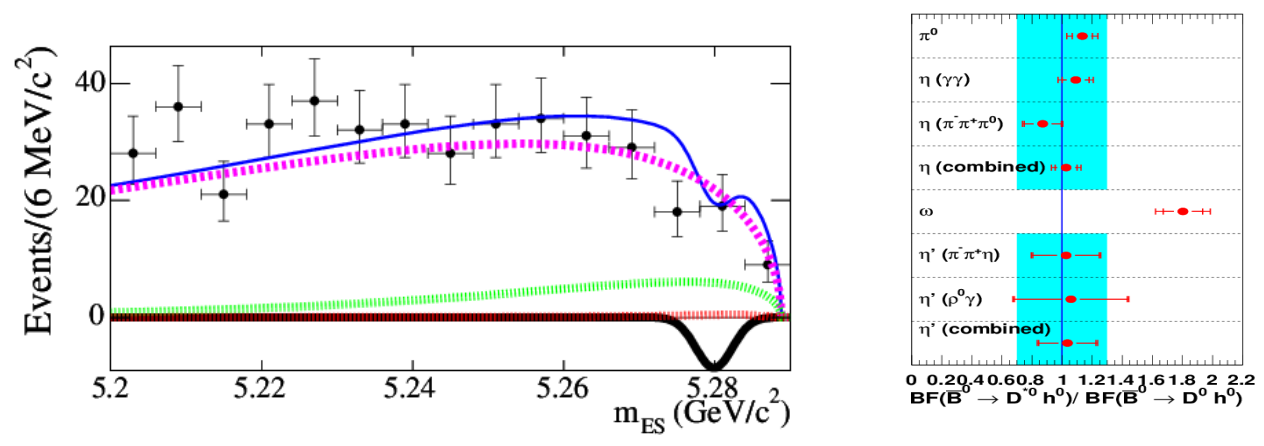

Figure 1: Left: projection plot on the $m_{\mathrm{ES}}$ variable of the ML fit extracting the signal yield for the channel $D^{+} K_{S}^{0}$, with $D^{+} \rightarrow K^{-} \pi^{+} \pi^{+}$. The black solid line represents the signal component, the magenta dotted line is the $B \bar{B}$ combinatorial, the green dotted is the continuum, and the red dotted is the peaking background: no signal is found. Right: ratio $\mathscr{B}\left(B \rightarrow D^{* 0} h^{0}\right) / \mathscr{B}\left(B \rightarrow D^{0} h^{0}\right)$ for the $B^{0} \rightarrow D^{(*)} h^{0}$ analysis. The results confirm the predictions by SCET.

\section{Final states containing baryons}

$B$ meson decays with baryons in the final state account for $\sim 7 \%$ of the total, but the sum of the currently measured modes reaches only $\sim 1 \%$. These decays are poorly described by the theory, so more experimental information can be useful to refine the phenomenological models. In several cases enhancements at threshold or discrepancies with respect to the predictions based on phase-space arguments have been observed.

We report the first observation of the decay $\bar{B}^{0} \rightarrow \Lambda_{c} \bar{p} \pi^{0}$ [4]. Signal candidates are searched for by combining $\bar{p}$ and $\pi^{0}$ candidates with a $\Lambda_{c} \rightarrow p K^{-} \pi^{+}$candidate. A ML fit to the $m_{\mathrm{ES}}$ distributions extracts a signal yield of $273 \pm 23$ events, which corresponds to $\mathscr{B}\left(\bar{B}^{0} \rightarrow \Lambda_{c} \bar{p} \pi^{0}\right)=(1.94 \pm 0.17 \pm$ $0.14 \pm 0.50) \times 10^{-4}$, where the first quoted uncertainty is the statistical error, the second is the systematic, and the third arises from the uncertainty on the branching fraction of the $\Lambda_{c}$ decay used in this measurement. We observe (see Figure 2) an enhancement at the threshold of the invariant mass of the $\Lambda_{c} \bar{p}$ system. Finally, we find no evidence of the $\bar{B}^{0} \rightarrow \Sigma_{c} \bar{p}$ decay. 

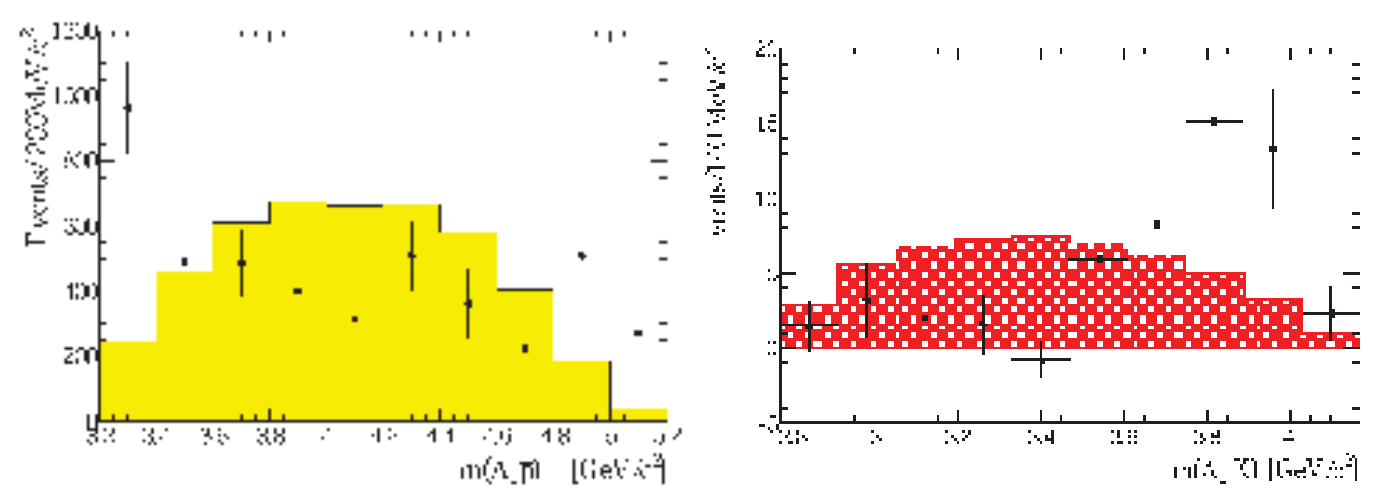

Figure 2: Left plot: invariant mass of the $\Lambda_{c} \bar{p}$ in the $\bar{B}^{0} \rightarrow \Lambda_{c} \bar{p} \pi^{0}$ analysis. The points with error bars represent the data, the histogram is the simulation generated according to the phase-space assumption; a clear enhancement at threshold is visible. Right: invariant mass of the $\Lambda_{c} K^{-}$in the $\bar{B}^{0} \rightarrow \Lambda_{c} \bar{\Lambda} K^{-}$analysis. The points with error bars represent the data, while the histogram is the phase-space Monte Carlo: a yet to be explained structure is visible at a mass of $\sim 3.9 \mathrm{GeV} / c^{2}$.

The decay $\bar{B}^{0} \rightarrow \Lambda_{c} \bar{\Lambda} K^{-}$is searched for using a strategy similar to the analysis presented above. The decays $\bar{B}^{0} \rightarrow \Lambda_{c} \bar{p} \pi^{+} K^{-}$, having the same final state of the mode under investigation, constitute a source of peaking background, which is suppressed imposing a cut on the flight-length of the $\bar{\Lambda}$ candidate. The signal is extracted with a ML fit to the $\Delta E$ variable and yields $51 \pm 9$ events, which correspond to a branching fraction $\mathscr{B}\left(\bar{B}^{0} \rightarrow \Lambda_{c} \bar{\Lambda} K^{-}\right)=(3.8 \pm 0.9 \pm 0.2 \pm 1.0) \times 10^{-5}$ [5], where the first error is statistical, the second is systematic, and the third derives from the uncertainty on the $\Lambda_{c} \rightarrow p K^{-} \pi^{+}$branching fraction. The analysis of the invariant mass of the $\Lambda_{c} K^{-}$system, shows a structure around $3.9 \mathrm{GeV} / \mathrm{c}^{2}$ (see Figure 2) which is inconsistent with the phase-space MC and is not explained by any known resonance.

\section{Conclusions}

After more than three years from the end of the data-taking the BABAR Collaboration continues to exploit its rich dataset. The latest measurements on hadronic $B$ meson decays provide new constraints for the existence of New Physics contributions and will help theorists in the development of more reliable phenomenological models.

\section{References}

[1] BABAR Collaboration, P. del Amo Sanchez et al., Phys. Rev. D 82, 092006 (2010).

[2] BABAR Collaboration, P. del Amo Sanchez et al., Phys. Rev. D 83, 032004 (2011).

[3] BABAR Collaboration, J. P. Lees et al., arXiv:1107.5751 [hep-ex], submitted to Phys. Rev. D.

[4] BABAR Collaboration, B. Aubert et al., Phys. Rev. D 82, 031102(R) (2011).

[5] BABAR Collaboration, J. P. Lees et al., arXiv:1108.3211 [hep-ex], submitted to Phys. Rev. D. 